\title{
Perfuração Cardíaca Durante Cateterismo em um Lactente com Estenose Valvar Pulmonar: Resultados Satisfatórios Pela Abordagem Híbrida
}

\author{
Carlos Augusto Cardoso Pedra ${ }^{1}$, Santiago Raul Arrieta ${ }^{1}$, Lucidia Rodrigues Costa ${ }^{1}$, \\ Antonio Flavio Sanches de Almeida
}

\section{RESUMO}

Relatamos o caso de um lactente de 10 meses, submetido a uma tentativa mal sucedida de valvoplastia pulmonar com balão (VPB) devido à perfuração cardíaca. A criança foi subseqüentemente tratada por esternotomia mediana, sutura do sítio de perfuração no átrio direito e VPB intraoperatória por meio de uma bainha colocada na via de saída do ventrículo direito.

DESCRITORES: Estenose da valva pulmonar. Dilatação com balão. Lactente.
A valvoplastia pulmonar com balão (VPB) é o método de escolha para tratar a estenose valvar pulmonar (EVP), sendo considerado um dos procedimentos intervencionistas pediátricos mais comuns e seguros no laboratório de cateterismo'. Entretanto, a perfuração cardíaca pode ocorrer durante a manipulação do cateter ao tentar-se alcançar a artéria pulmonar, especialmente em lactentes ${ }^{2,3}$. Nos últimos anos, a abordagem híbrida foi proposta para tratar alguns defeitos congênitos do coração, tais como comunicações ventriculares (CIV), em lactentes ${ }^{4-7}$, e a síndrome do coração esquerdo hipoplásico (SCEH), em neonatos ${ }^{8}$. Nesse relato, descrevemos um caso em que ocorreu perfuração cardíaca durante o cateterismo de um lactente, que seria submetido a VPB. Após drenagem percutânea do espaço pericárdico, o local da perfuração foi suturado através de uma toracotomia mediana e a válvula dilatada com um cateter-balão avançado através de uma

\footnotetext{
Instituto Dante Pazzanese de Cardiologia, São Paulo, SP. Correspondência: Carlos Augusto Cardoso Pedra. Av. Dr. Dante Pazzanese, 500 - São Paulo - SP - Brasil - CEP 04012-180 Tel: (11) 5085-6114 - Fax: (11) 5085-6196 E.mail: cacpedra@uol.com.br

Recebido em: 29/5/2007 • Aceito: 5/7/2007
}

SUMMARY

Cardiac Perforation During Catheterization in an Infant with Pulmonary Valve Stenosis: Successful Outcomes by a Hybrid Approach

We report a case in which a 10-month-old infant underwent an unsuccessful attempt at percutaneous pulmonary balloon valvuloplasty (PBV) due to cardiac perforation. The child was subsequently managed by median sternotomy, suture of the bleeding site in the right atrium and intraoperative PBV through a sheath placed in the right ventricular outflow tract.

DESCRIPTORS: Pulmonary valve stenosis. Balloon dilatation. Infant.

bainha curta, colocada cirurgicamente na via de saída do ventrículo direito (VSVD).

\section{RELATO DO CASO}

Um paciente do sexo masculino, não dismórfico, com 10 meses (pesando 8,5 kg), com EVP foi encaminhado para nossa instituição para realização da VPB. Era assintomático e não recebia medicação. No exame físico, na radiografia do tórax e no eletrocardiograma, notaram-se os achados clássicos da EPV. A ecocardiografia transtorácica revelou hipertrofia moderada a importante do ventrículo direito, tamanho normal do anel pulmonar (medindo $11 \mathrm{~mm}$ ), espessamento e abertura em cúpula dos folhetos da valva pulmonar (VP) e gradiente instantâneo de pico através da VSVD de $96 \mathrm{mmHg}$. O tronco da artéria pulmonar e a artéria pulmonar esquerda (APE) estavam dilatados. A criança foi levada ao laboratório de cateterismo e, sob anestesia geral, o acesso vascular foi obtido com a colocação de uma bainha de $6 \mathrm{Fr}$ na veia femoral direita. Sulfato de heparina foi administrado (100 UI/kg). As seguintes pressões (em $\mathrm{mmHg}$ ) foram registradas: Átrio direito: 10; Ventrículo direito: 100/12. A pressão arterial avaliada não invasivamente foi de 90/50 mmHg. Um ventriculograma direito confirmou os achados ecocardiográficos, 
Pedra CAC, et al. Perfuração Cardíaca Durante Cateterismo em um Lactente com Estenose Valvar Pulmonar: Resultados Satisfatórios Pela Abordagem Híbrida. Rev Bras Cardiol Invas 2007; 15(3): 293-296.

mostrando os sinais clássicos da EVP (Figura 1). Tentouse cateterizar a VSVD com um cateter Judkins de coronária direita 5Fr (Cordis Corporation, Miami, FL) e avançar um fio-guia hidrofílico de 0,035" (Glidewire; Terumo Cardiovascular Systems Corporation, Ann Harbor, MI) para a extremidade distal da artéria pulmonar esquerda (APE). Neste momento, notou-se que o fio-guia apresentava um caminho incomum ao redor da silhueta cardíaca. O fio foi retirado e um pouco de contraste foi injetado através do cateter, causando opacificação do espaço pericárdico (Figura 2). Como havia aumento progressivo da silhueta cardíaca com halo duplo notado na fluoroscopia, realizou-se a drenagem percutânea do espaço pericárdico com a técnica Marfan, antes que ocorresse deterioração hemodinâmica. O derrame pericárdico foi subseqüentemente confirmado pelo ecocardiografia. Embora a heparina tenha sido completamente neutralizada, aspirava-se sangue não coagulável continuadamente do espaço pericárdico, sendo injetado no braço lateral da bainha venosa femoral. Como o sangramento pericárdico não diminuiu após 40-45 minutos, decidiu-se submeter a criança a uma toracotomia exploradora mediana, para identificar e suturar o sítio da perfuração. A criança foi levada ao centro cirúrgico e preparada de maneira usual. Após a abertura do pericárdio, uma perfuração minúscula foi notada na ponta do apêndice atrial direito. Esta foi suturada com fio de Prolene (Ethicon, Somerville, NJ) em pontos contínuos. O sangramento foi imediatamente controlado, com a criança permanecendo em excelente condição hemodinâmica. Decidiu-se, então, realizar a VPB no centro cirúrgico. A VSVD foi puncionada com um abocath e um fio hidrofílico 0,035" (Terumo) foi avançado até a extremidade distal de APE. Uma bainha curta de $7 \mathrm{Fr}$ foi colocada na VSVD sobre o fio e fixada com uma sutura em bolsa com fio Prolene (Ethicon), como descrito em outras séries ${ }^{4}$. O fio hidrofílico foi trocado por um fio teflonado comum (0,035"; $150 \mathrm{~cm}$ ) (Cook Cardiology, Bloomington, IN) e um cateter-balão Tyshak (Numed, Cornwall, ON, Canadá) $15 \mathrm{~mm} \times 3 \mathrm{~cm}$ foi colocado através da bainha, no coração pulsando. Medidas foram feitas entre o anel da VP e o sítio de punção da VSVD, para estimar a posição adequada do balão através da VP antes da sua inserção. O balão foi parcialmente insuflado de forma manual com soro fisiológico e sua posição adequada foi confirmada pela palpação do anel pulmonar. A insuflação completa foi realizada duas vezes e a estabilização do balão foi conseguida de forma habitual. O cateter-balão foi retirado e a pressão intra-operatória (em $\mathrm{mmHg}$ ) foi medida com uma agulha: tronco da artéria pulmonar: 25/15; VSVD: 38/10. A bainha foi removida e a VSVD suturada e fechada como de costume. A criança teve uma recuperação sem problemas, recebendo alta após 5 dias. Ecocardiograma transtorácico antes da alta revelou abertura satisfatória dos folhetos da VP, insuficiência pulmonar discreta e gradiente instantâneo máximo de $25 \mathrm{mmHg}$ através da VSVD. Após dois anos de acompanhamento, o paciente permaneceu assintomático e ecocardiogramas repetidos mostraram gradientes máximos variando de 35 a $55 \mathrm{mmHg}$.

\section{DISCUSSÃO}

Com o desenvolvimento tecnológico dos cateteres e aperfeiçoamento das técnicas hemodinâmicas, a perfuração cardíaca tornou-se uma complicação rara durante o cateterismo diagnóstico e intervencionista em

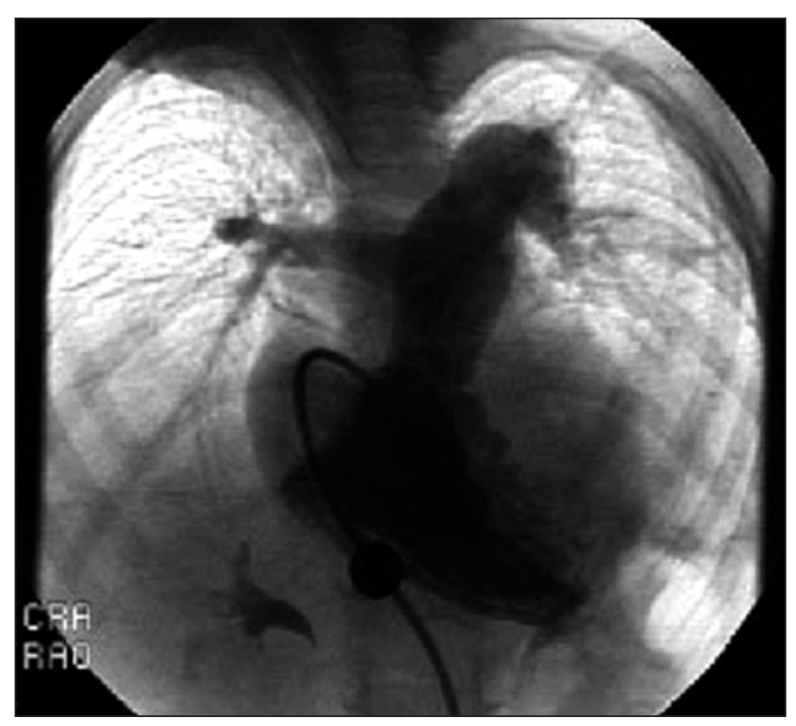

Figura 1 - Ventriculograma direito na projeção oblíqua anterior direita com inclinação cranial, mostrando hipertrofia ventricular direita significativa, tamanho normal do anel pulmonar (11-12 mm) e espessamento e abertura em cúpula dos folhetos da valva pulmonar. O tronco da artéria pulmonar e a artéria pulmonar esquerda estavam notadamente dilatados.

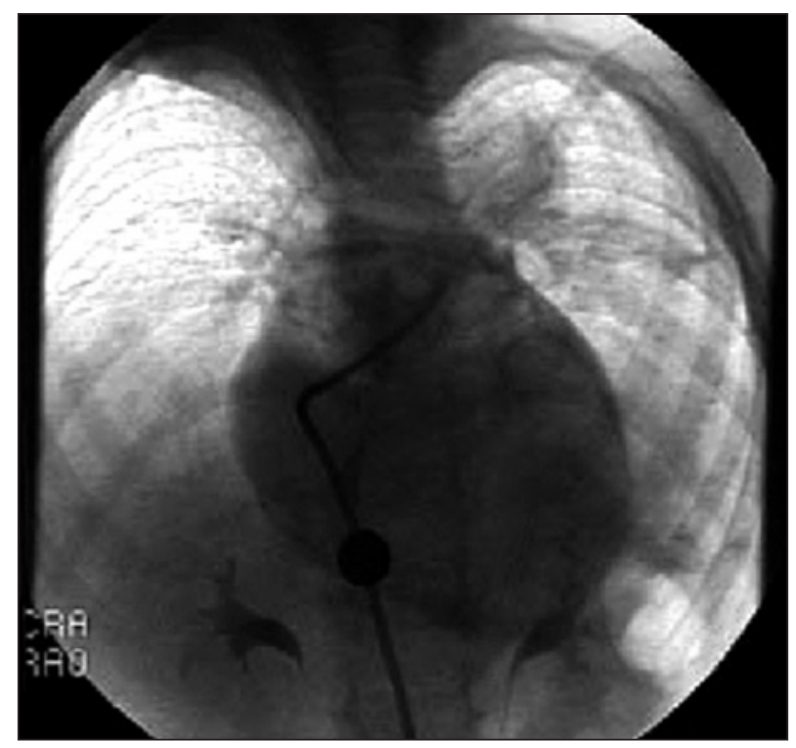

Figura 2 - Injeção de uma pequena quantidade de contraste através de um cateter Judkins de artéria coronária direita, mostrando opacificação do espaço pericárdico. 
Pedra CAC, et al. Perfuração Cardíaca Durante Cateterismo em um Lactente com Estenose Valvar Pulmonar: Resultados Satisfatórios Pela Abordagem Híbrida. Rev Bras Cardiol Invas 2007; 15(3): 293-296.

crianças $^{9,10}$. Entretanto, na VPB para EVP em neonatos e lactentes, a perfuração cardíaca pode ocorrer durante a manipulação do cateter na VSVD na tentativa de avançar o fio-guia até a extremidade distal da artéria pulmonar $^{2,3}$. O local de perfuração é geralmente localizado na VSVD, mas também pode ser encontrado no apêndice atrial direito. Este último local é particularmente susceptível a rasgos e perfurações devido a sua fina espessura e friabilidade, especialmente em lactentes como este aqui apresentado. Portanto, cuidado extremo e técnica judiciosa devem ser praticados para evitar esta complicação. Se a perfuração ocorrer, é crucial que seu reconhecimento seja imediato para evitar o tamponamento cardíaco e instabilidade hemodinâmica. No caso citado, isso foi possível reparando no trajeto incomum do fio ao redor da silhueta cardíaca e a ampliação desta com formação de um duplo halo no fluoroscópio. O diagnóstico de derrame pericárdico deve ser confirmado pela ecocardiografia. A drenagem pericárdica previne o tamponamento e deve ser realizada imediatamente por profissionais experientes, no laboratório de cateterismo. Uma vez que o espaço pericárdico é drenado e o sangue retorna à circulação, deve-se esperar um tempo para verificar se o sangramento cessa espontaneamente. Isso não é incomum quando fios finos (0,014") são empregados e envolvidos na perfuração ${ }^{11,12}$. Entretanto, quando a perfuração é causada pelo próprio cateter ou quando ele é avançado sobre o fioguia para o espaço pericárdico, o sangramento contínuo é a regra e, usualmente, requer exploração cirúrgica.

Neste caso, após o local da perfuração ter sido identificado e suturado através de uma esternotomia mediana, duas opções cirúrgicas foram consideradas para tratar a VP estenótica: a valvotomia pulmonar através da VSVD pela técnica de Brock e a valvotomia aberta com circulação extracorpórea ${ }^{13}$. A primeira opção foi empregada satisfatoriamente na década de 50, embora possa ser associada com sangramento significativo. Além disto, pode não fornecer alívio adequado da estenose em alguns casos. A segunda opção é associada aos inconvenientes da circulação extracorpórea. Baseados nas publicações recentes sobre abordagens híbridas para fechar CIVs em lactentes ${ }^{4-7}$, como também paliar a SCEH em neonatos ${ }^{8}$, este tipo de procedimento emergiu como uma terceira opção neste caso. A VPB intra-operatória provou ser factível, segura e eficaz, tendo sido provavelmente a melhor escolha naquela situação, evitando a circulação extracorpórea e diminuindo a necessidade de transfusão de sangue. Entretanto, alguns comentários devem ser discutidos a respeito da técnica usada. A inserção da bainha na VSVD foi realizada de maneira similar a publicações anteriores $^{4}$. Apesar de alguns procedimentos híbridos previamente descritos terem sido realizados sob monitoração ecocardiográfica e/ou fluoroscópica, nenhum destes métodos estava disponível em nosso centro cirúrgico. Mesmo assim, achamos que o avanço do fio-guia até a extremidade distal da APE não foi difícil, pois o vaso possuía grosso calibre e sua orientação espacial funcionava como uma quase continuação da VSVD e do tronco da artéria pulmonar. Percebemos, também, que o posicionamento adequado do cateter-balão não apresentou problemas, pois este era facilmente palpável pela VP após realizar as medidas corretas. A insuflação do balão e o manuseio do cateter foram realizados conforme o habitual. As medidas diretas da pressão para avaliar os resultados hemodinâmicos foram obtidas da mesma maneira empregada após a correção da tetralogia de Fallot ${ }^{14}$. Neste sentido, alguns centros baseiam-se somente nos dados hemodinâmicos para avaliar os resultados imediatos da VPB, não sendo realizados angiogramas de forma rotineira após a dilatação (Jim Lock, comunicação pessoal). O ecocardiograma transtorácico pós-operatório confirmou uma dilatação efetiva. Baseado na elevação discreta do gradiente instantâneo de pico em vários exames seqüenciais, uma nova dilatação talvez seja realizada futuramente.

Em conclusão, a VPB intra-operatória pode ser realizada de forma segura e eficaz em pacientes com EVP que necessitem de esternotomia para controle do sangramento pericárdico devido à perfuração cardíaca durante o cateterismo cardíaco.

\section{REFERÊNCIAS BIBLIOGRÁFICAS}

1. Pihkala J, Nykanen D, Freedom RM, Benson LN. Interventional cardiac catheterization. Pediatr Clin North Am. 1999;46(2): 441-64.

2. Zeevi B, Keane JF, Fellows KE, Lock JE. Balloon dilation of critical pulmonary stenosis in the first week of life. J Am Coll Cardiol. 1988;11(4):821-4.

3. Gildein HP, Kleinert S, Goh TH, Wilkinson JL. Treatment of critical pulmonary valve stenosis by balloon dilatation in the neonate. Am Heart J. 1996;131(5):1007-11.

4. Amin Z, Danford DA, Lof J, Duncan KF, Froemming S. Intraoperative device closure of perimembranous ventricular septal defects without cardiopulmonary bypass: preliminary results with the perventricular technique. J Thorac Cardiovasc Surg. 2004;127(1):234-41.

5. Amin Z, Berry JM, Foker JE, Rocchini AP, Bass JL. Intraoperative closure of muscular ventricular septal defect in a canine model and application of the technique in a baby. J Thorac Cardiovasc Surg. 1998;115(6):1374-6.

6. Maheshwari S, Suresh PV, Bansal M, Mishra A, Sharma R, Amin Z. Perventricular device closure of muscular ventricular septal defects on the beating heart. Indian Heart J. 2004;56(4):333-5.

7. Bacha EA, Cao QL, Starr JP, Waight D, Ebeid MR, Hijazi ZM Perventricular device closure of muscular ventricular septal defects on the beating heart: technique and results. J Thorac Cardiovasc Surg. 2003;126(6):1718-23.

8. Galantowicz M, Cheatham JP. Fontan completion without surgery. Semin Thorac Cardiovasc Surg Pediatr Card Surg Annu. 2004;7:48-55.

9. Cassidy SC, Schmidt KG, Van Hare GF, Stanger P, Teitel DF. Complications of pediatric cardiac catheterization: a 3-year study. J Am Coll Cardiol. 1992;19(6):1285-93.

10. Vitiello R, McCrindle BW, Nykanen D, Freedom RM, Benson LN. Complications associated with pediatric cardiac catheterization. J Am Coll Cardiol. 1998;32(5):1433-40. 
Pedra CAC, et al. Perfuração Cardíaca Durante Cateterismo em um Lactente com Estenose Valvar Pulmonar: Resultados Satisfatórios Pela Abordagem Híbrida. Rev Bras Cardiol Invas 2007; 15(3): 293-296.

11. Pedra CA, de Sousa LN, Pedra SR, Ferreira WP, Braga SL, Esteves CA, et al. New percutaneous techniques for perforating the pulmonary valve in pulmonary atresia with intact ventricular septum. Arq Bras Cardiol. 2001;77(5):471-86.

12. Humpl T, Soderberg B, McCrindle BW, Nykanen DG, Freedom RM, Williams WG, et al. Percutaneous balloon valvotomy in pulmonary atresia with intact ventricular septum: impact on patient care. Circulation. 2003;108(7):826-32.

13. Kouchoukos NT, Blackstone E, Doty DB, Hanley FL, Karp
RB. Pulmonary stenosis and intact ventricular septum. In: Kouchoukos NT, Blackstone E, Doty DB, Hanley FL, Karp RB, eds. Kirklin/ Barrat-Boyes cardiac surgery. Philadelphia: Churchill Livingstone;2003. p.1075-94.

14. Kouchoukos NT, Blackstone E, Doty DB, Hanley FL, Karp RB. Ventricular septal defect with pulmonary stenosis or atresia. In: Kouchoukos NT, Blackstone E, Doty DB, Hanley FL, Karp RB, eds. Kirklin/ Barrat-Boyes cardiac surgery. Philadelphia: Churchill Livingstone;2003. p.1946-2074. 